\title{
Group velocity and energy transport velocity near the band edge of a disordered coupled cavity waveguide: an analytical approach
}

\author{
N. Le Thomas* and R. Houdré \\ Institut de Physique de la Matière Condensée, École Polytechnique Fédérale de Lausanne (EPFL), Station 3, \\ CH-1015 Lausanne, Switzerland \\ *Corresponding author: nicolas.lethomas@epfl.ch
}

Received May 27, 2010; revised August 17, 2010; accepted August 20, 2010; posted August 23, 2010 (Doc. ID 129119); published September 22, 2010

\begin{abstract}
We develop an analytical approach to theoretically investigate light speed propagation near the band edge of a coupled cavity waveguide in the presence of residual disorder. This approach that is based on a mean field theory allows us to define the domains of validity of the group velocity and the energy transport velocity concepts as well as a guideline to minimize the role of the residual disorder. Inspired by an analogy with the theory of multiple scattering of classical wave, we derive an analytical formula for the energy transport velocity in periodic photonic structures. Whereas the group velocity diverges near the band edge in the presence of any amount of residual disorder, we show that the energy transport velocity mainly follows the ideal group velocity of the unperturbed structure except for very strong disturbances out of the scope of the presented model. (C) 2010 Optical Society of America

OCIS codes: $130.2790,130.5296,230.4555,260.2030,290.4210,310.6628$.
\end{abstract}

\section{INTRODUCTION}

Slowing down the light, which naturally propagates at $c$ $\approx 3 \times 10^{8} \mathrm{~m} / \mathrm{s}$ in vacuum, is currently under strong investigation in integrated photonic structures [1,2]. This was in particular motivated by the experimental demonstration of light pulses propagating at the speed of a bicycle in atomic gas [3]. The slowing down of the light speed is commonly understood as a decrease in the group velocity that is defined as $v_{g}=\mathrm{d} \omega / \mathrm{d} k[4,5]$, where $\omega$ is the angular frequency, and $k$ is the spatial wave number. The group velocity denotes the speed propagation of the center of a wave packet [6]. This concept is convenient to describe the propagation of a bit of information, but becomes less relevant in the vicinity of dielectric constant resonances [7]. Although $v_{g}$ keeps a physical meaning near a resonance for very specific conditions, such as a small sample thickness to avoid any distortion of the pulse [8-10], it cannot be associated with the speed of the energy transport. In addition, all the effects of advance or delay of a pulse observed near a resonance are limited by the initial temporal pulse width, because such effects only result from a reshaping of the pulse instead from a significant rigid temporal shift of all the envelope of the pulse [8,9].

In periodic non-absorbing dielectric structures, the socalled slow light regime is reached by engineering flat dispersion curves $\omega(k)$ [2], such as, for instance, in photonic crystal coupled cavity waveguide (CCW) structures $[11,12]$, or more generally in plasmonic structures [13], and in metamaterials $[14,15]$. Similarly to slowdown systems that rely on electromagnetic induced transparency $[3,16]$, the coupled resonator structures exhibit a welldefined dispersion curve with an associated delay of the pulse that is not directly linked and limited by the tem- poral width of the pulse itself. It is crucial to point out that the decrease in speed of a light pulse based on such an approach takes place with no loss of information: the energy carried by all the frequencies that constitute the pulse is conserved during the propagation, as long as the pulse bandwidth is smaller than the pass band of the medium. In contrast, a rate of energy loss induced by absorption or out-of-plane scattering, in the case of planar structures, or a filtering of some of the frequency of the pulse by an optical resonator, for instance, can be experimentally interpreted as an ultra-slowing down of the transport of information [2]. However, in both of these cases the price to pay is a loss of a large amount of information. That is why such kinds of processes will be ignored here.

Real photonic structures are always subject to residual disorder inherent to state-of-the-art technological fabrication. Such a disorder is the source of multiple-scattering effects that profoundly modify the nature of the transmission $[17,18]$ as well as the dispersion curve $\omega(k)[19,20]$. In a similar way as the physical meaning of the group velocity is modified near a resonance of the medium dielectric constant, the question of the impact of the disorder on the group velocity and in general on the speed of light in periodic photonic structures can be raised. In particular, we have recently reported an abrupt transition between a well-defined $\omega(k)$ relation (dispersive regime) and a speckle-like dispersive diagram (diffusive regime) in single line defect photonic crystal waveguides [21]. Such an experimental observation highlights the qualitative difference between the group velocity $v_{g}$ and the energy transport velocity $v_{E}$.

In the current paper we present an analytical model based on a standard mean field theory to explore the tran- 
sition between the group velocity $v_{g}$ and the energy transport velocity $v_{E}$ in periodic photonic structures subject to a uniform residual disorder. Our aim is to discuss, within the framework of this model, the robustness and the nature of the slow light regime when a residual disorder comes into play. The starting point is a textbook calculation of a one-dimensional (1D) dispersion curve that is based on a standard tight binding approximation and that can be used to describe generic CCWs. Depending on the amount of residual disorder, we use this ideal model to link together the shape of the dispersion curve and the frequency band where $v_{g}$ loses its meaning in favor of $v_{E}$. First, we outline the fundamental hypotheses of the analytical model. In the second part we determine the dispersion diagram of a coupled cavity structure according to the amount of residual disorder. As a result, we derive the group velocity of a disordered structure and propose one strategy to overcome the detrimental impact of residual disorder. We pursue in the third part the calculation of the energy transport velocity. In the last part, we discuss and summarize the main results of the model and comment on the light matter interaction in the diffusive transport regime. Sections 2 and 5, which are selfcontained, can be both read separately in order to get a comprehensive overview of the main results without entering the details of the calculations.

\section{GENERAL FORMULATION OF THE MODEL AND ITS HYPOTHESES}

We consider an infinite 1D periodic system formed by identical coupled photonic cavities. A classical scalar field that satisfies the Helmholtz equation describes the wave propagation. The eigenstates of the coupled system can be expanded into different bases: either extended Bloch wave functions or linear combinations of Wannier functions localized at the individual cavities. We use the tight binding approximation, i.e., we suppose that the Wannier functions can be approximated by the modes of the single cavities, which are referred to as "atomic" states below. This approximation holds if the fields of neighboring cavities overlap weakly enough. Within this assumption of a linear combination of cavity modes, the dispersion curve is given by $(\omega(k) / c)^{2}=\left(\omega_{m} / c\right)^{2}+2 T \cos (k \Lambda)=\left(\omega_{0} / c\right)^{2}$, with $\omega_{m}$ being the mean frequency of the band, $T$ the transfer integral, and $\Lambda$ the period of the 1D chain. Such a dispersion curve restricted to one half of the first Brillouin zone is plotted in Fig. 1(a) (dark line), where we have defined the normalized frequency $x$ as $x=\left(\left(\omega_{0} / c\right)^{2}-\left(\omega_{m} / c\right)^{2}\right) / 2 T$. We assume a single atomic state per cavity, and as a result a single frequency band is formed. The width of the band, $\Delta \omega=4 T c$, is directly related to the coupling strength between the states localized at each cavity. Note that the normalized frequency $x$ can be written $x=\left(u-u_{m}\right) /(\Delta u / 2)$, with $u=(\Lambda / 2 \pi)(\omega / c)$ being the reduced frequency, $u_{m}$ $=(\Lambda / 2 \pi)\left(\omega_{m} / c\right)$ the central reduced frequency, and $\Delta u$ the bandwidth. The dispersion curves as well as the group and the energy transport velocities will be expressed in terms of $x$. We do not consider the effect of pure dissipation such as out-of-plane scattering in planar photonic structures, which are discussed in [22].

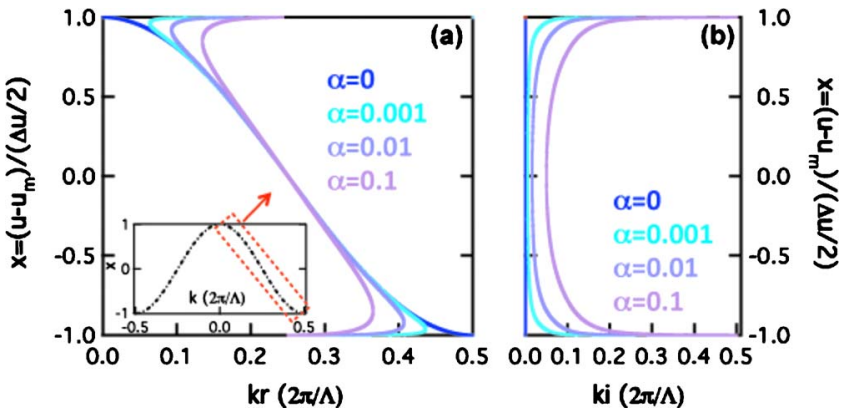

Fig. 1. (Color online) Dispersion curves of a coupled cavity structure subject to residual disorder for (a) the real part of the wave vector within the positive part of the first Brillouin zone and (b) the imaginary part of the wave vector. Inset: Dispersion curve over the entire first Brillouin zone. The $\alpha$ parameter quantifies the amount of disorder, and $x$ is the normalized band frequency (see text). $\Lambda$ is the lattice constant of the coupled cavity structure. $u_{m}$ and $\Delta u$ : bandwidth and frequency at the middle of the band, respectively.

We use a perturbative approach whose starting point is the Green's function of an ideal coupled cavity system. Three kinds of perturbation can be induced by the residual disorder of the dielectric map: (1) When averaged over several configurations, the disorder does not allow one to distinguish the different cavities and induces an inhomogeneous linewidth broadening of the single cavities that is smaller enough than the coupling frequency between the cavities; (2) if the disorder is not homogeneous or follows special statistics laws such as the Levi distribution [23], its averaging over several configurations inside each of the cavities is such that peak frequencies of each atomic states are different; and (3) the disorderinduced linewidth broadening of the atomic states is larger than the coupling frequency between the cavities. The two latter cases are out of the scope of our model. Case (2) can correspond to a disorder whose one of the characteristic length scales is larger than the period of the cavity chain; for instance, there are some isolated and rare defects along the chain that do not follow the expected Gaussian distribution of a sub-nanometer residual disorder. Case (3) implies that the strength of the disorder is too strong to consider a perturbative approach. In the first case, the averaging procedure of the supposed homogeneous residual disorder allows one to recover the invariance per translation of the coupled cavity chain. For one realization of disorder, the eigenfrequency of each atom of the coupled cavity chain is different. Averaging over several configurations of disorder results in an inhomogeneous broadening that is identical for all atoms. Note that reaching experimentally a situation corresponding to the first case implies a well controlled technological processing.

\section{ANALYTICAL DISPERSION CURVE OF A DISORDERED COUPLED CAVITY STRUCTURE}

In the following part, we detail the analytical calculation of the dispersion curve presented in Fig. 1. The ideal dispersion curve of the coupled cavity structure, $(\omega(k) / c)^{2}$ $=\left(\omega_{m} / c\right)^{2}+2 T \cos (k \Lambda)=\left(\omega_{0} / c\right)^{2}$, is the starting point of our 
1D perturbation approach. The corresponding 1D Green's function of the unperturbed Helmholtz equation, i.e., the field produced at $r$ by a point source located at $r^{\prime}$, is given by [24]

$$
G^{0}\left(r, r^{\prime} ; k_{0}^{2}\right)=-\frac{1}{T} \frac{\rho_{1}^{|l-m|}}{\rho_{1}-\rho_{2}}=\frac{1}{T} \frac{\left(x-\sqrt{x^{2}-1}\right)^{|l-m|}}{2 \sqrt{x^{2}-1}},
$$

with $k_{0}=\omega_{0} / c$ being the free space wave number, $r=l \Lambda$ and $r^{\prime}=m \Lambda, l$ and $m$ integers, and $\rho_{1}=x-\sqrt{x^{2}-1}$ and $\rho_{2}$ $=x+\sqrt{x^{2}-1}$. Note that we have assumed in Eq. (1) that the size of the field of one cavity state is approximately equal to the periodicity $\Lambda$ of the coupled cavity chain. In order to stay in a general framework, we consider that the periodicity $\Lambda$, or equivalently here the size of the cavity mode, and the transfer integral $T$ are independent parameters: it means that the size of the single cavity field and the coupling strength are decoupled.

With the presence of disorder and for a harmonic point source, the field satisfies the Helmholtz equation $\Delta \Psi(\omega ; r)+k_{0}^{2}\left(n_{0}^{2}(\omega ; r)+\mu(\omega ; r)\right) \Psi(\omega ; r)=\delta(r)$, where $n_{0}(\omega ; r)$ is the phase index of the unperturbed structure, and $\mu(r)$ is the fluctuating part of the dielectric constant resulting from the disorder. We assume that the fluctuating part $\mu(r)$ is a centered homogeneous random function whose correlation function is expressed as $\left\langle\mu(r) \mu\left(r^{\prime}\right)\right\rangle=\epsilon^{2} \exp (\mid r$ $\left.-r^{\prime} \mid / \sigma\right)=\Gamma_{\mu}\left(\left|r-r^{\prime}\right|\right)$, with $\sigma$ being the correlation length and $\Gamma_{\mu}$ the correlation function. The function $\Gamma_{\mu}$ completely characterizes the statistic of $\mu(r)$, as only the twopoint correlation function is non-vanishing for a centered Gaussian random function.

Due to the statistical nature of the problem, our aim is to find the first moment of the field, $\left\langle G\left(r, r^{\prime} ; k_{0}^{2}\right)\right\rangle$, also called the mean Green's function, and its second moment or covariance $\left\langle G\left(r, r^{\prime} ; k_{0}^{2}\right) \bar{G}\left(\rho, \rho^{\prime} ; k_{0}^{2}\right)\right\rangle$, where $\langle\cdots\rangle$ means averaging over different configurations, and the overbar stands for complex conjugate. The poles of the Fourier transform $\left\langle\hat{G}\left(k ; k_{0}^{2}\right)\right\rangle$ of the mean Green's function provide the dispersion diagram of the disordered structure [24], whereas the second moment will be used to determine the energy transport velocity in the diffusive regime. Based on a diagrammatic method [25], $\left\langle\hat{G}\left(k ; k_{0}^{2}\right)\right\rangle$ can be formally determined with the Dyson equation as

$$
\left\langle\hat{G}\left(k ; k_{0}^{2}\right)\right\rangle=\frac{1}{\left[\hat{G}\left(k ; k_{0}^{2}\right)\right]^{-1}-\hat{\Sigma}\left(k ; k_{0}^{2}\right)},
$$

where the self-energy $\hat{\Sigma}\left(k ; k_{0}^{2}\right)$ is the sum of all connected diagrams, and

$$
\left\langle\hat{G}^{0}\left(k ; k_{0}^{2}\right)\right\rangle=F T\left\{G^{0}\left(\left|r-r^{\prime}\right| ; k_{0}^{2}\right)\right\}=\frac{1}{k_{0}^{2}-\frac{\omega_{m}^{2}}{c^{2}}+2 T \cos (k \Lambda)},
$$

where $F T\{\cdots\}$ stands for Fourier transform. Within this procedure, the determination of the dispersion curve consists merely of calculating the self-energy. By retaining only the first term of the self-energy, the so-called first- order smoothing approximation [25], the self-energy takes a simple form:

$$
\hat{\Sigma}\left(k ; k_{0}^{2}\right)=\epsilon^{2} k_{0}^{4} F T\left\{G^{(0)}\left(\left|r-r^{\prime}\right|\right) \Gamma_{\mu}\left(\left|r-r^{\prime}\right|\right)\right\} .
$$

The analytical calculation of this last expression gives

$$
\begin{aligned}
\hat{\Sigma}\left(k ; k_{0}^{2}\right)= & \frac{\epsilon^{2}}{T} \frac{k_{0}^{4}}{\rho_{2}-\rho_{1}} \frac{1}{\Lambda} \frac{2\left(1 / \sigma-i\left(\theta_{1} / \Lambda\right)\right)}{\left(1 / \sigma-i\left(\theta_{1} / \Lambda\right)\right)^{2}+k^{2}} \text { for }|x|<1, \\
\hat{\Sigma}\left(k ; k_{0}^{2}\right)= & \frac{\epsilon^{2}}{T} \frac{k_{0}^{4}}{\rho_{2}-\rho_{1}} \frac{1}{\Lambda} \frac{2\left(1 / \sigma+\ln \left|\rho_{1}\right| / \Lambda-i\left(\theta_{1} / \Lambda\right)\right)}{\left(1 / \sigma+\ln \left|\rho_{1}\right| / \Lambda-i\left(\theta_{1} / \Lambda\right)\right)^{2}+k^{2}} \\
& \text { for }|x|>1,
\end{aligned}
$$

where $\theta_{1}=\arg \left(\rho_{1}\right)$ with the assumption that the disorder correlation length is negligible compared to the size of the cell, i.e., $\sigma \ll \Lambda$, these expressions can be further simplified as

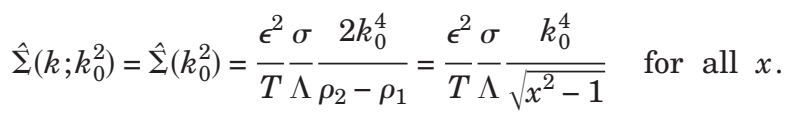

Such a hypothesis implies that the impact of the disorder is the same on average for each cell of the coupled system, i.e., all the cells are identically perturbed on average, and the inhomogeneous broadening of their spectra resulting from the averaging procedure is identical, which is in agreement with the framework that we have previously defined for the current model. From the analytical expression of the self-energy, the equation that defines the dispersion curves, i.e., $\left[\hat{G}\left(k ; k_{0}^{2}\right)\right]^{-1}-\hat{\Sigma}\left(k ; k_{0}^{2}\right)=0$, takes the following simple form:

$$
x-\cos (k \Lambda)-\frac{\sqrt{\alpha}}{\sqrt{x^{2}-1}}=0,
$$

with $\sqrt{\alpha}=\left(\epsilon^{2} \sigma / 2 T^{2} \Lambda\right) k_{0}^{2}$. This last parameter contains the characteristic parameters $\epsilon$ and $\sigma$ of the model of the disorder that was chosen. Moreover, we assume that the bandwidth $(\Delta \omega / c)=4 T$ is much smaller than the middle frequency $\omega_{m} / c$ of the band, which allows us to approximate $k_{0}^{4} \approx\left(\omega_{m} / c\right)^{4}$ and to include this term in the control parameter $\alpha$.

The determination of the dispersion curve consists of finding the explicit relation between $x$ and $k$ for different $\alpha$ 's. For $\alpha \neq 0$ and for real $x$, only complex $k$ 's are solutions of the previous equation. By writing $k=k_{r}+i k_{i}$, we obtain in particular for $|x|<1 \cos \left(\Lambda k_{r}\right)=x / \sqrt{y}$ and $\operatorname{ch}\left(\Lambda k_{i}\right)=x / \sqrt{y}$, with

$$
y=\frac{1}{2}\left[x^{2}+1+\frac{\alpha}{1-x^{2}}+\sqrt{\left(x^{2}+1+\frac{\alpha}{1-x^{2}}\right)-4 x^{2}}\right] .
$$

The corresponding dispersion curves $x\left(k_{r}\right)$ and $x\left(k_{i}\right)$ for different $\alpha$ 's are plotted in Figs. 1(a) and 1(b), respectively. The maximum value of the $\alpha$ parameter has been chosen to match recent experimental dispersion curves $x\left(k_{r}\right)$ of photonic crystal CCWs that were reported in $[12,22]$. Such a value fits data from state-of-the-art deep 
ultraviolet or electron-beam lithography patterning. Whereas the band edges are profoundly modified by an increasing amount of disorder quantified by $\alpha$, the central half-part of the band remains almost unperturbed for $x\left(k_{r}\right)$. In this part of the dispersion curve, the main effect of the disorder results in an increase in $k_{i}$ as shown in Fig. 1(b). The imaginary part of the wave number corresponds to a damping of the first moment of the field. When averaged over different configurations of disorder, the corresponding angular spectrum of the field looks like a smooth broad peak whose linewidth is given by $2 k_{i}$ (inhomogeneous broadening). In practice, only a single configuration of disorder can be measured in a particular sample, and the resulting angular spectrum has a speckle-like shape whose envelope corresponds to the averaged case. Note that a spatial averaging resulting from an infinite length of the structure is not equivalent to an averaging over several configurations of disorder due to the damping of the field.

Considering that $\alpha \propto 1 /\left(T^{2} \Lambda\right)^{2}$ and that the plots in Fig. 1(a) are given in normalized coordinates, the independence of the central part of the band on the parameter $\alpha$ is true whatever is the value of the bandwidth, provided that the $T^{2} \Lambda$ product is constant. Based on the assumption of the current model, this implies that as small as wanted $v_{g}$ can be reached in the middle of the band if the coupling strength $T$, or equivalently $\Delta u$, decreases, and $T^{2} \Lambda$ is constant. As long as the hypothesis developed in Section 2 is satisfied, in particular as long as the different cells that form the coupled structure cannot be distinguished by the residual disorder on average, i.e., an averaging over different configurations of the disorder in each cell, there are no theoretical intrinsic limits for achieving vanishing group velocities. In practice, however, the increase in $\Lambda$ that is imposed by the condition $T^{2} \Lambda=$ const sets a severe constraint on the quality of the technological fabrication due to the requirement of a homogeneous disorder over all the cells: a 1 decade decrease in $T$, or equivalently of $v_{g}$, requires a 2 decade increase in $\Lambda$, and consequently of the sample size.

Although the validity of the definition of $v_{g}$ in the middle of the band does not raise a major issue for the state-of-the-art amount of residual disorder, Fig. 1(a) reveals that at the band edge the curvature of the dispersion curve is dramatically modified. It implies that $v_{g}$ increases, or concomitantly the group index $n_{g}$ decreases, in contrary to the intended slowing down effect and finally even diverges. Such a behavior appeals for another definition of the speed of light in the same spirit as in [7].

\section{MICROSCOPIC DETERMINATION OF THE SPEED OF ENERGY TRANSPORT IN THE DIFFUSIVE REGIME}

When the dispersive contribution of the intensity transport becomes negligible compared with the diffusive contribution, the energy transport can be described with the classical diffusion equation [21]. For such a regime of undefined group velocity, van Tiggelen et al. developed a microscopic approach of the multiple scattering in random dielectric particles in order to determine the macroscopic diffusion coefficient and in particular the speed of the en- ergy transport from $a b$ initio parameters [26]. Their approach consists of solving the Bethe-Salpeter equation for the second moment of the field. Here, we highlight the formal similarity between the mean Green's function associated with the problem of the light propagation through random point scatterers and the one of the light propagation in simple periodic structures with a sinusoidal dispersion curve. We take advantage of this correspondence to give an analytical expression for the energy transport velocity for different values of the disorder parameter $\alpha$.

For a system of random isotropic point scatterers the mean Green's function is given by [25]

$$
\langle\hat{G}(\omega ; k)\rangle=\frac{1}{(\omega / c)^{2}-k^{2}-\hat{\Sigma}(\omega)},
$$

with $\hat{\Sigma}(\omega)=n t(\omega)$, and where $n$ is the density of scatterers and $t(\omega)$ is the scattering factor. The corresponding energy transport velocity $v_{E}$ is a function of $n t(\omega)$. For the coupled cavity system the mean Green's function can be written as

$$
\langle\hat{G}(\omega ; k)\rangle=\frac{1}{(\omega / c)^{2}-\left(\omega_{m} / c\right)^{2}-2 T \cos (k \Lambda)-\hat{\Sigma}(\omega)},
$$

with $\hat{\Sigma}(\omega)=\hat{\Sigma}\left(k_{0}^{2}\right)=\sqrt{\alpha} / \sqrt{x^{2}-1}$. Near the band edge that is the focus of our current study, the " $\cos (k \Lambda)$ " function can be developed to the second order, and then

$$
\langle\hat{G}(\omega ; k)\rangle \approx \frac{1}{(\omega / c)^{2}-k^{2}\left(T \Lambda^{2}\right)-\left(\omega_{m} / c\right)^{2}+2 T-\hat{\Sigma}(\omega)} .
$$

This last expression allows a formal correspondence with the standard point scattering problem if $n t(\omega)=\hat{\Sigma}(\omega)$ is replaced with $n t(\omega)=\left(\omega_{m} / c\right)^{2}-2 T+\hat{\Sigma}(\omega)$, where $\hat{\Sigma}(\omega)$ is a pure complex number here. The comparison between the mean Green's functions of the two models is possible as long as the development of the $\cos (k \Lambda)$ term is a good approximation. To estimate the validity range of the previous approximate development, the resulting ideal group velocity, i.e., without disorder, is plotted as a dashed line curve in Fig. 2. By comparing this curve with the corresponding exact result obtained in the previous section, we see that such an approximation is satisfactory for $x>0.7$. Note that in Section 3 we have determined the dispersion curve and as a result the group velocity without any series expansion for the $\cos (k \Lambda)$ term. Here we take advantage of this expansion to simplify the calculation of the energy transport velocity. Without any resonance effect, i.e., in the present case far from the band edge, the energy transport velocity is expected to equal the unperturbed group velocity. We will show that, here, $v_{E}$ significantly deviates from this standard value only for $x>0.9$.

With the use of the ladder approximation [27] and of the Fourier-Laplace transform $L(q, \Omega)$ of the intensity scattered by one scattering point, the Bethe-Salpeter equation can be conveniently transformed into [28] $L(q, \Omega)=S(q, \Omega) /[1-M(q, \Omega)]$, with $S(q, \Omega)$ being a source term, and the kernel 


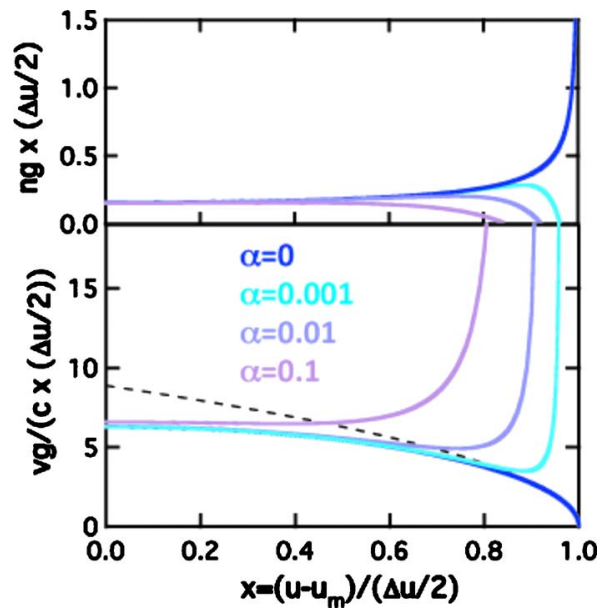

Fig. 2. (Color online) Variations of the group index $n_{g}$ (top) and of the group velocity $v_{g}$ (bottom) of a wave propagating in a coupled cavity structure according to the normalized band frequency $x$ for different amounts of residual disorder ( $c$ : speed of light). The group velocity and the group index are normalized with respect to the bandwidth $\Delta u$. Dashed line: group velocity resulting from a second order approximation of the ideal dispersion curve.

$$
M(q, \Omega)=n \bar{t}\left(\omega_{-}\right) t\left(\omega_{+}\right) \int G\left(p_{+}, \omega_{+}\right) \bar{G}\left(p_{-}, \omega_{-}\right) \frac{\mathrm{d} p}{(2 \pi)^{d}}
$$

with $d$ being the dimension of the problem, $p_{ \pm}=p \pm \frac{1}{2} q$, and $\omega_{ \pm}=\omega \pm \frac{1}{2} \Omega$. The parameters $\Omega$ and $q$ represent the slow varying parameters of the light pulse, whereas $p$ and $\omega$ are the fast variables, which involve one period of the wave. To determine the kernel, the Fourier-Laplace transform of the mean Green's function is expanded to first order in $\Omega$ and in second order in $q$ as describe in $[28,29]$, for instance. As a result of the particular dispersion curve of the unperturbed structure, the coefficients that appear in the formula of the kernel are different from the ones obtained in [28] with the standard random scatterers model. We find in the case of the coupled cavity chain

$$
M(q, \Omega)=1-\frac{1}{d} l^{2} q^{2}-i \Omega \tau
$$

where $l=k_{e} / \operatorname{Im}(n t(\omega))=(1 / \sqrt{\alpha})\left(k_{0} / 2 T\right)\left(v_{g}^{\mathrm{eff}} / c\right) \sqrt{1-x^{2}}$ is the scattering mean free path, and $v_{g}^{\text {eff }}=\mathrm{d} \omega / \mathrm{d} k_{e}$ is the effective group velocity for which we have defined $k_{e}$ $=\sqrt{k_{0}^{2}-\operatorname{Re}(n t(\omega))}$. As the real part of the scattering factor does not depend on $\omega$, this effective velocity becomes equal to the unperturbed group velocity: $v_{g}^{\mathrm{eff}}=c\left(k_{e} / k_{0}\right)$ $=c\left(\sqrt{2 T} / k_{0}\right) \sqrt{1-x}=v_{g 0}$. The time $\tau$ is the sum of two contributions [30]: $\tau=\left(k_{0} / k_{e}\right) \tau_{s c}+\tau_{d w}$, with the scattering time $\tau_{s c}=l / c$ and the dwell time $\tau_{d w}$. The dwell time is associated with the ability of resonances to store the energy of the field at a given frequency, such as Mie resonances in the case of powder of spherical scatterers [26]. From the determination of the kernel, the following expression is obtained for the dwell time in the case of the standard random scatterers model [28]:

$$
\tau_{d w}=\operatorname{Im}\left(\frac{1}{t} \frac{\mathrm{d} t}{\mathrm{~d} \omega}\right)+\frac{2 \pi}{k_{e} \bar{t} t} \operatorname{Re}\left(\frac{\mathrm{d} t}{\mathrm{~d} \omega}\right) .
$$

The same expression is obtained in the case of the CCW within our approximate development of the $\cos (k \Lambda)$ term. For the CCW the scattering factor is given by

$$
n t(\omega)=\left(\omega_{m} / c\right)^{2}-2 T-\frac{\sqrt{\alpha}}{\sqrt{x^{2}-1}} .
$$

Hence its real part is constant. This implies the simplification $\tau_{d w}=\operatorname{Im}[(1 / t)(\mathrm{d} t / \mathrm{d} \omega)]$ and finally leads to the following analytical formula:

$$
\frac{\tau_{d w}}{\tau_{s c}}=\frac{c}{v_{g 0}} \frac{2 x}{\left(1-x^{2}\right)} \frac{\alpha u_{m} \Delta u}{u_{m}^{2}\left(1-x^{2}\right)+\alpha \Delta u^{2}} .
$$

From the expression of the kernel obtained above, the energy transport velocity is defined by $v_{E}=l / \tau$. This can be rewritten in the analytical form

$$
v_{E}=\frac{v_{g 0}}{1+\frac{v_{g 0}}{c} \frac{\tau_{d w}}{\tau_{s c}}}=v_{g 0} \frac{1}{1+\frac{2 x}{\left(1-x^{2}\right)} \frac{\alpha u_{m} \Delta u}{u_{m}^{2}\left(1-x^{2}\right)+\alpha \Delta u^{2}}} .
$$

In Fig. 3 we show the variation of $v_{E}$ near the band edge for different $\alpha$ 's. Over more than $90 \%$ of the band $v_{E}$ can be approximated with the group velocity of the ideal structure, and in comparison to the group velocity, $v_{E}$ is relatively unaffected by the parameter $\alpha$. The contribution of the dwell time resulting from the multiplescattering processes becomes significant only for frequencies located in the last $4 \%$ of the band, i.e., for frequencies quite far from the frequency where $v_{g}$ becomes undefined. In practice the contribution of the dwell time can be difficult to observe due to weak or strong localization effects [31-35]. Note that the calculation of the dwell time relies on the ladder approximation that misses contributions in the development of the irreducible kernel such as crosseddiagrams. The formula obtained for the velocity transport is valid only in the diffusive regime; in particular it is inappropriate for sub-diffusive and anomalous diffusion. Nevertheless the energy velocity transport is expected to vanish, in contrast to the group velocity that is subject to a lower bound near the band edge due to the residual disorder.

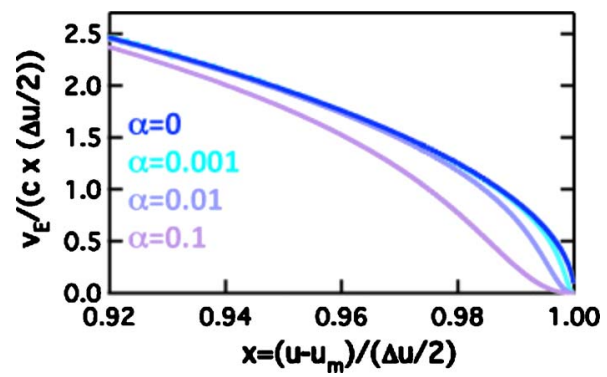

Fig. 3. (Color online) Variation of the energy transport velocity $v_{E}$ of a wave propagating in a coupled cavity structure near the band edge for different amounts of residual disorder quantified by the $\alpha$ parameter (see text). 


\section{DISCUSSION ON SPEED OF LIGHT AT THE BAND EDGE OF A DISORDERED COUPLED CAVITY STRUCTURE}

In Fig. 4, we highlight the dispersion curve near the top band edge of a generic coupled cavity structure subject to residual disorder and focus on the corresponding group and energy velocities for a given amount of disorder. The disorder is quantified by the single characteristic constant $\alpha$ that has been previously defined above. The velocities are normalized with respect to the speed of light in vacuum $c$ and to $\Delta u / 2$, in order to provide a universal plot whatever is the coupling strength between the cavities. The purpose of this universal plot is to provide a comprehensive link between the velocity of a wave that propagates in a disordered coupled cavity structure and the shape of the dispersion curve.

For normalized frequencies $x$ below a value of 0.7 , which depends on $\alpha$, the main difference between the unperturbed dispersion curve $(\alpha=0)$, represented as a dotted-dashed line, and the perturbed one (here $\alpha=0.01$ ), lies in the linewidth broadening of the spectral response of the structure. Such a broadening is highlighted with the gray area that corresponds to the spectral linewidth at half-maximum along the wave number axis. Above $x$ $=0.7$, the dispersion curve subject to disorder starts to differ significantly from the ideal case, in line also with a large broadening of the spectral response. In parallel, from $x=0.7$, the group velocity $v_{g}$ does not decrease toward zero as expected and begins to diverge with a horizontal asymptote labeled $L_{1}$. In contrast, the energy velocity $v_{E}$ strictly follows the variation of the group velocity $v_{g 0}$ of the corresponding ideal structure, except at the extreme parts of the band edges or for an amount of disorder that is out of the range of the model.

As recently observed in $[21,22]$ for a given realization of disorder, the dispersion curve suddenly stops to be defined well before the excitation frequency reaches the band edge. This effect results in a transition between a single broadened spectral peak and a speckle-like angular field distribution. As a consequence the group velocity looses its meaning after such a transition. Within the current averaged ensemble model, the $L_{1}$ asymptote puts an upper limit for the range of validity of $v_{g}$, which gives an

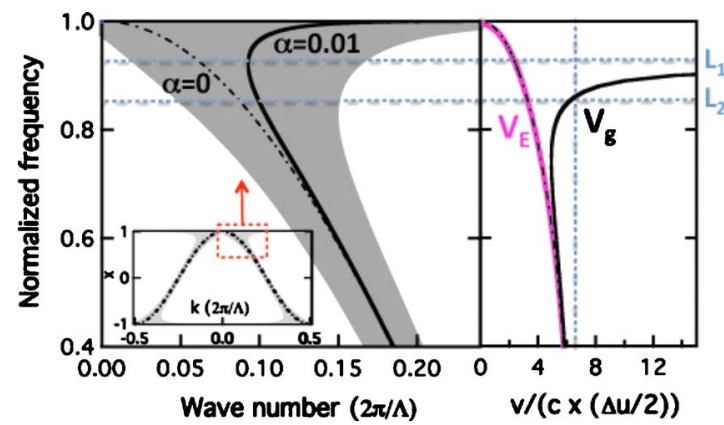

Fig. 4. (Color online) Left: Universal dispersion curve of a CCW near the band edge without ( $\alpha=0$, dashed-dotted line) and with ( $\alpha=0.01$, plain line) disorder. Right: Corresponding group velocity $v_{g}$ and energy transport velocity $v_{E}(\Lambda, c, \Delta u$ : lattice constant, speed of light, and bandwidth, respectively). Gray shadow: inhomogeneous linewidth broadening at half-maximum. Inset: Dispersion curve over the entire first Brillouin zone. interpretation of the experimental observation reported in $[21,22]$. Nevertheless, a second characteristic quantity, labeled $L_{2}$, can be defined in the framework of our model: it corresponds to the value where $v_{g}$ exceeds its value at the middle of the band.

As discussed in [21], an undefined dispersion relation does not preclude the energy transport of a pulse. In terms of multiple-scattering theory, the intensity of the wave that propagates can be separated into two contributions [27,36]. The speed of propagation of the first contribution is given by $v_{g}$, whereas for the second contribution it is $v_{E}$. The relative weight between these two contributions provides a criterion to define the actual speed of light to be considered for a propagating wave packet. While the first contribution is described by a ballistic transport equation, the second contribution is governed by a diffusion equation as shown below. As the first contribution is exponentially attenuated along the propagation with a damping factor equal to $1 / k_{i}$, this contribution will be significant only on a length scale smaller than $1 / k_{i}$, which corresponds to around six cavities for $\alpha=0.01$.

Considering that the dispersive regime, i.e., the region of the band where the group velocity exists [21], allows a propagation of the information of the field in a welldefined basis, it is the preferred regime to transport the information of a pulsed wave. However, the slow transport velocity in the diffusive regime could be advantageously used to enhance nonlinear effects, in particular the Kerr effect, as the pulse spends more time at a given place of the photonic structure. When the group velocity is meaningful, the spatial compression of the pulse inherent to the slowing down effect [1] is expected to enhance the nonlinear Kerr interaction, as long as the dispersion curve is not modified. In the diffusive regime the situation is more complex. Depending on the sign of the Kerr coefficient enhanced spreading of the pulse (defocusing case) or wave collapse (focusing case) has been recently discussed in [37]. In the first case we expect an increase in $v_{E}$ (super-diffusive regime), whereas in the second case $v_{E}$ should decrease (sub-diffusive regime). We would like to stress that nonlinear effects profoundly modify the role played by the disorder as, for instance, simulated in [38] with a mechanical model based on a linear chain of masses linked by springs with a quadratic nonlinearity. In particular the nonlinearity can lead to an effective smoothing of the disordered dielectric potential, in analogy to the transition from Bose-glass phase to superfluid phase as predicted in the Bose gas [39]. The dispersion curve of a coupled cavity structure can also be drastically transformed due to nonlinear effects in the same way as it is renormalized by the presence of pure dissipation [19] or as it is flipped according to the first diagonal of the dispersion diagram in distributed feedback lasers with strong gain modulation [40].

\section{CONCLUSION}

To conclude, we have developed an analytical model that describes the dispersive properties of coupled cavity structures and in particular the speed of light in such structures. In the middle of the frequency band, this 
model predicts that the group velocity does not significantly depend on the amount of disorder and as a result can be as small as wanted provided that the residual disorder is homogeneous over all the structure, which is challenging for the current state-of-the-art technology. At the band edges, the speed of light is strongly altered by the disorder-induced multi-scattering processes and can only be defined as an energy transport velocity. According to the analytical formula that we have derived in the framework of a mean field theory, the energy transport velocity varies as the ideal group velocity of the unperturbed structure and can be vanishingly small, as long as the wave transport is in a diffusive regime.

\section{ACKNOWLEDGMENTS}

This project was supported by the Swiss NCCR-Quantum Photonics, the Swiss National Science Foundation (SNSF) (project 200021-124413), and the COST action MP0702.

\section{REFERENCES}

1. T. F. Krauss, "Slow light in photonic crystal waveguides," J. Phys. D 40, 2666-2670 (2007).

2. T. Baba, "Slow light in photonic crystals," Nat. Photonics $\mathbf{2}$, 465-473 (2008).

3. L. V. Hau, S. E. Harris, Z. Dutton, and C. H. Behroozi, "Light speed reduction to 17 meters per second in an ultracold atomic gas," Nature 397, 594-598 (1999).

4. M. Born and E. Wolf, Principles of Optics, 7th ed. (Cambridge U. Press, 1999).

5. J. D. Jackson, Classical Electrodynamics, 3rd ed. (Wiley, 1998).

6. D. Bohm, Quantum Theory (Prentice-Hall, 1966).

7. L. Brillouin, Wave Propagation and Group Velocity (Academic, 1960).

8. C. G. B. Garrett and D. E. McCumber, "Propagation of a Gaussian light pulse through an anomalous dispersion medium," Phys. Rev. A 1, 305-313 (1970).

9. S. Chu and S. Wong, "Linear pulse propagation in an absorbing medium," Phys. Rev. Lett. 48, 738-741 (1982).

10. J. Peatross, S. A. Glasgow, and M. Ware, "Average energy flow of optical pulses in dispersive media," Phys. Rev. Lett. 84, 2370-2373 (2000).

11. M. Notomi, E. Kuramochi, and T. Tanabe, "Large-scale arrays of ultrahigh- $Q$ coupled nanocavities," Nat. Photonics 2 , 741-747 (2008)

12. J. Jágerská, N. Le Thomas, V. Zabelin, R. Houdré, W. Bogaerts, P. Dumon, and R. Baets, "Experimental observation of slow mode dispersion in photonic crystal coupledcavity waveguides," Opt. Lett. 34, 359-361 (2009).

13. S. Zhang, D. A. Genov, Y. Wang, M. Liu, and X. Zhang, "Plasmon-induced transparency in metamaterials," Phys. Rev. Lett. 101, 047401 (2008).

14. P. Tassin, L. Zhang, Th. Koschny, E. N. Economou, and C. M. Soukoulis, "Low-loss metamaterials based on classical electromagnetically induced transparency," Phys. Rev. Lett. 102, 053901 (2009).

15. N. Liu, L. Langguth, T. Weiss, J. Kästel, M. Fleischhauer, T. Pfau, and H. Giessen, "Plasmonic analogue of electromagnetically induced transparency at the Drude damping limit," Nature Mater. 8, 758-762 (2009).

16. J. B. Khurgin, "Optical buffers based on slow light in electromagnetically induced transparent media and coupled resonator structures: comparative analysis," J. Opt. Soc. Am. B 22, 1062-1074 (2005).
17. S. Mazoyer, J. P. Hugonin, and P. Lalanne, "Disorderinduced multiple scattering in photonic-crystal waveguides," Phys. Rev. Lett. 103, 063903 (2009).

18. M. Patterson, S. Hughes, S. Combrié, N.-V.-Quynh Tran, A. De Rossi, R. Gabet, and Y. Jaouën, "Disorder-induced coherent scattering in slow-light photonic crystal waveguides," Phys. Rev. Lett. 102, 253903 (2009).

19. N. Le Thomas, V. Zabelin, R. Houdré, M. V. Kotlyar, and T. F. Krauss, "Influence of residual disorder on the anticrossing of Bloch modes probed in $k$ space," Phys. Rev. B 78, 125301 (2008).

20. S. Mookherjea and A. Oh, "Effect of disorder on slow light velocity in optical slow-wave structures," Opt. Lett. 32, 289-291 (2007).

21. N. Le Thomas, H. Zhang, J. Jágerská, V. Zabelin, and R. Houdré, "Light transport regimes in slow light photonic crystal waveguides," Phys. Rev. B 80, 125332 (2009).

22. J. Jágerská, H. Zhang, N. Le Thomas, and R. Houdré, "Radiation loss of photonic crystal coupled-cavity waveguides," Appl. Phys. Lett. 95, 111105 (2009)

23. J.-P. Bouchaud and A. Georges, "Anomalous diffusion in disordered media: Statistical mechanisms, models and physical applications," Phys. Rep. 195, 127-293 (1990).

24. E. N. Economou, Green's Functions in Quantum Physics, 2nd ed. (Springer-Verlag, 1979).

25. U. Frish, "Wave propagation in random media," in Probabilistic Methods in Applied Mathematics, A. T. BharuchaReid, ed. (Academic, 1968), Vol. 1, pp. 75-198.

26. B. A. van Tiggelen, A. Lagendijk, M. P. van Albada, and A. Tip, "Speed of light in random media," Phys. Rev. B 45, 12233-12243 (1992).

27. E. Akkermans and G. Montambaux, Mesoscopic Physics of Electrons and Photons (Cambridge U. Press, 2006).

28. M. C. W. van Rossum and T. M. Nieuwenhuizen, "Multiple scattering of classical waves: microscopy, mesoscopy, and diffusion," Rev. Mod. Phys. 71, 313-371 (1999).

29. P. Sheng, Introduction to Wave Scattering, Localization, and Mesoscopic Phenomena (Academic, 1995).

30. A. Lagendijk and B. A. van Tiggelen, "Resonant multiple scattering of light," Phys. Rep. 270, 143-215 (1996).

31. K. M. Yoo, F. Liu, and R. R. Alfano, "When does the diffusion approximation fail to describe photon transport in random media," Phys. Rev. Lett. 64, 2647-2650 (1990).

32. P. E. Wolf and G. Maret, "Weak localization and coherent backscattering of photons in disordered media," Phys. Rev. Lett. 55, 2696-2699 (1985).

33. Z. Q. Zhang, A. A. Chabanov, S. K. Cheung, C. H. Wong, and A. Z. Genack, "Dynamics of localized waves: Pulsed microwave transmissions in quasi-one-dimensional media," Phys. Rev. B 79, 144203 (2009).

34. S. John, "Strong localization of photons in certain disordered dielectric superlattices," Phys. Rev. Lett. 58, 24862489 (1987).

35. S. John, "Localization of light," Phys. Today 44(5), 32-40 (1991).

36. A. Ishimaru, Wave Propagation and Scattering in Random Media (Academic, 1978), Vols. 1 and 2.

37. G. Schwiete and A. M. Finkel'stein, "Non linear wavepacket dynamics in a disordered medium," Phys. Rev. Lett. 104, 103904 (2010).

38. O. Richoux, C. Depollier, and J. Hardy, "Propagation of mechanical waves in a one-dimensional nonlinear disordered lattice," Phys. Rev. E 73, 026611 (2006).

39. L. Fontanesi, M. Wouters, and V. Savona, "Superfluid to Bose-glass transition in 1D weakly interacting Bose gas," Phys. Rev. Lett. 103, 030403 (2009).

40. F. K. Kneubühl, in Theories on Distributed Feedback Laser, Vol. 14 of Laser Science and Technology, V. S. Letokhov, C. V. Shank, Y. R. Shen, and H. Walther, eds. (Hardwood Academic, 1993), p. 18. 